\title{
Duodenal pseudotumour: a silent impacted common bile duct calculus
}

\author{
P.J. Finch, J. Haddock and A. Grundy
}

Departments of Medicine and Diagnostic Radiology, St George's Hospital, London, UK.

\begin{abstract}
Summary: We report the case of a man with mild dyspeptic symptoms referred for barium meal, who was initially thought to have a duodenal tumour. Subsequent investigation showed this to be a pseudotumour caused by impaction of a gallstone in the distal common bile duct, and endoscopic sphincterotomy effected a cure. This readily treatable condition should be remembered when filling defects are demonstrated in the duodenal loop.
\end{abstract}

\section{Introduction}

Impaction of calculi in the distal common bile duct commonly gives rise to biliary colic, obstructive jaundice or ascending cholangitis, and may precipitate acute pancreatitis. We report the case of a man in whom a mass in the medial wall of the second part of the duodenum was found during investigation for non-specific dyspepsia. Endoscopic retrograde cholangiopancreatography (ERCP) revealed the cause to be an impacted calculus in the common bile duct, and endoscopic sphincterotomy successfully dealt with the problem.

\section{Case report}

A 61 year old man presented with a one-year history of vague intermittent epigastric discomfort, present on three occasions in the previous 6 weeks. The pain did not radiate and there were no precipitating factors or associated jaundice or biliary colic. Serum alkaline phosphatase, bilirubin and alanine transaminase were all within the reference ranges. The patient was referred by his general practitioner for a double contrast barium meal.

This demonstrated a smooth rounded filling defect on the medial aspect of the second part of the duodenum with no evidence of ulceration or deformity of the duodenal loop (Figure 1). Endoscopy was subsequently performed and confirmed a smooth filling defect in the second part of the duodenum without mucosal ulceration. A superficial biopsy

Correspondence: P.J. Finch, M.D., M.R.C.P., Department of Medicine II, St. George's Hospital Medical School, Cranmer Terrace, London SW17 ORE, UK.

Accepted: 7 April 1989 revealed normal duodenal mucosa. An ultrasound examination demonstrated a dilated common bile duct measuring $2 \mathrm{~cm}$ in diameter although no calculi were seen and the pancreas appeared normal.

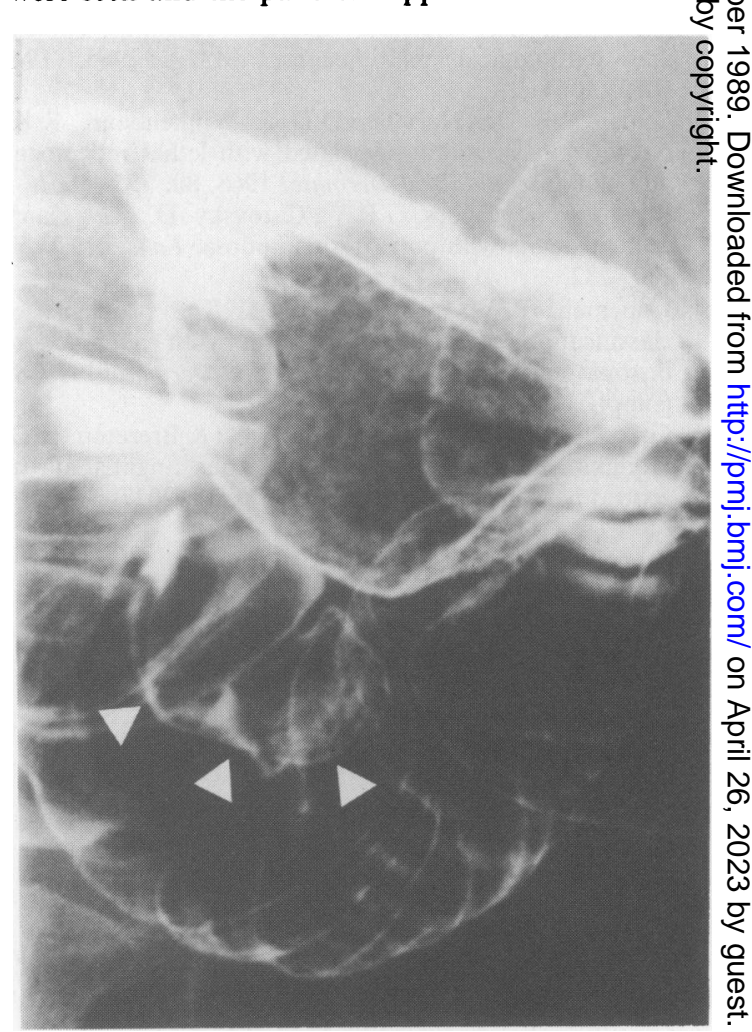

Figure 1 Double contrast barium examination of the duodenal loop. Smooth filling defect (arrowed).

(C) The Fellowship of Postgraduate Medicine, $1989 \stackrel{\mathbb{\complement}}{\varrho}$ 
The patient underwent ERCP when the smooth mass in the medial wall of the duodenum was again noted. The ampulla of Vater was found at its inferior margin. Cannulation of the common bile duct and injection of contrast medium revealed a large single calculus at the lower end of the common bile duct with proximal dilatation of the bile duct (Figure 2). The contrast injection disimpacted the calculus (Figure 3), but the mass on the medial wall of the second part of the duodenum persisted and probably represented soft tissue oedema around the lower end of the common bile duct. A sphincterotomy was performed and the calculus successfully removed with a Dormia basket.

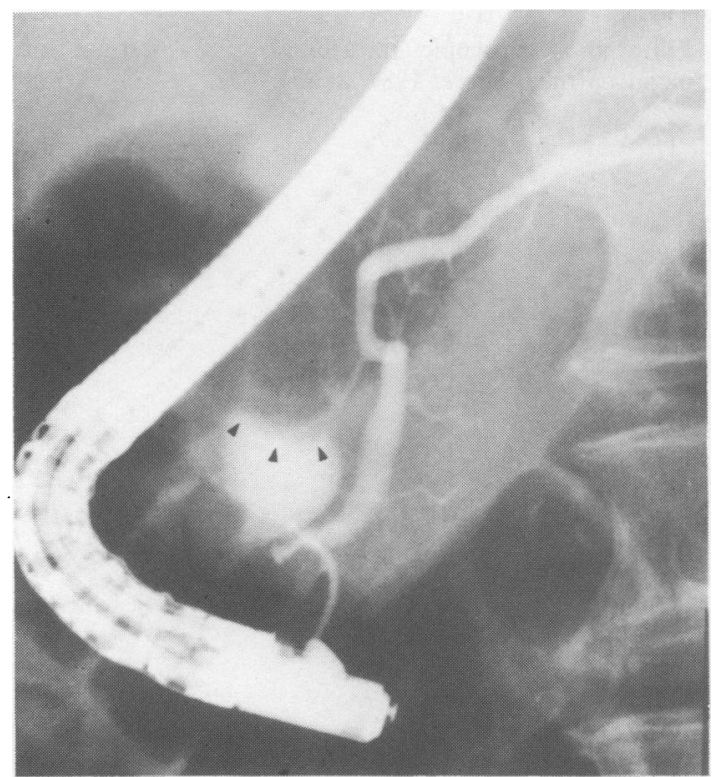

Figure 2 Calculus (arrowed) impacted in the distal common bile duct.

\section{Discussion}

The differential diagnosis of a mass lesion on the medial aspect of the descending duodenum includes a primary lesion in the duodenal wall or ampulla of Vater, and pathology in the adjacent head of the pancreas. Both pancreatitis and carcinoma of the head of pancreas may cause an impression on the medial aspect of the duodenum. A primary tumour arising from the duodenal wall, such as a leiomyoma, lipoma, carcinoid tumour or primary carcinoma of the duodenum should also be considered.

The mucosa overlying a leiomyoma is typically smooth with effacement of duodenal folds, sometimes

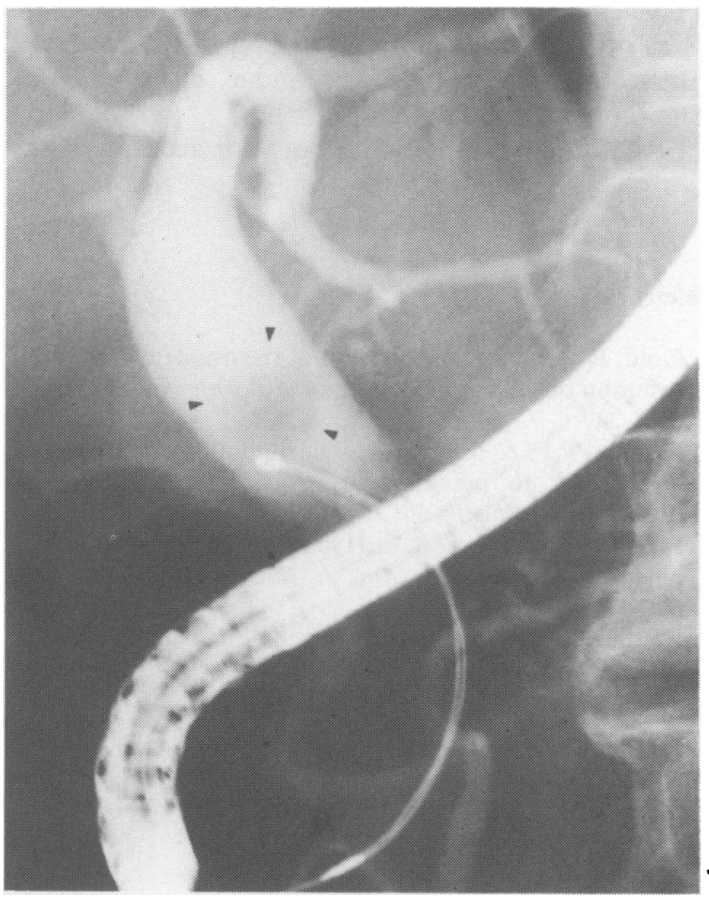

Figure 3 Calculus (arrowed) disimpacted prior to retrieval from the common bile duct with a basket.

with central ulceration. A primary carcinoma of the duodenum typically shows evidence of mucosal destruction. A small ampullary tumour or distal common bile duct tumour may also produce a mass effect in the duodenum and cause diagnostic confusion. ${ }^{1}$

The normal duodenal papilla varies considerably in size and may reach up to $3 \mathrm{~cm}$ in length, becoming more prominent when a gallstone becomes impacted, with subsequent oedema, and dilatation of the common bile duct above the calculus. The incidental finding of a calculus partially obstructing the common bile duct without producing any biliary symptoms, as in this case must be unusual.

In the past, barium studies have been found helpful in the assessment of biliary tract disease, ${ }^{2}$ and in particular obstructive jaundice, ${ }^{3,4}$ but have now been largely superseded by ultrasound, ERCP and computed tomography (CT). Ultrasound has a high accuracy in the detection of common bile duct calculi although occasionally non-echogenic calculi at the lower end of the common bile duct can be missed or misinterpreted as a pancreatic mass. ${ }^{5}$ CT has been suggested as being particularly useful in the assessment of neoplastic duodenal lesions, allowing accurate staging in $80 \%$ of malignant tumours. ${ }^{6}$ Although it is 
not yet generally available, endoscopic ultrasonography offers high resolution imaging of the pancreas without intervening bowel gas, and excellent mucosal definintion in the duodenum.?

ERCP offers the potential both for accurate diag-

\section{References}

1. Gold, R.P. Medial indentation of the duodenal sweep by common bile duct dilatation. Am J Roentgenol 1979, 133: 233-237.

2. Jacques, P.F. \& Bream, C.A. Barium duodenography as an adjunct to percutaneous transhepatic cholangiography. Am J Roentgenol 1978, 130: 693-696.

3. Bree, R.L. \& Flynn, R.E. Hypotonic duodenography in obstructive jaundice. Am J Roentgenol 1972, 116: 309-319.

4. Eaton, S.B., Ferrucci, J.T., Benedict, K.T. \& Margulis, A.F. Diagnosis of choledocholithiasis by barium duodenal examination. Radiology 1972, 102: 267-273. nosis and for therapy, and we suggest that a stone impacted at the lower end of the common bile duct should be considered, even in an asymptomatic patient, when a mass is found in the medial aspect of the second part of the duodenum.

5. Dewbury, K.C. \& Smith, C.L. The misdiagnosis of common bile duct stones with ultrasound. Br J Radiol 1983, 56: 625-630.

6. Farah, M.C., Jafri, S.Z.H. \& Schwab, R.E. et al. Duodenal neoplasms: role of CT. Radiology 1987, 162: 839-843.

7. Editorial. Endoscopic ultrasound - a marriage of inconvenience. Lancet 1987, ii: 431-432. 